\section{Count of B-lines: A Matter with Persistent Limitations}

\section{To the Editor:}

After reading the interesting review by Gutierrez, et al ${ }^{1}$, entitled "Ultrasound in the assessment of interstitial lung disease in systemic sclerosis. A systematic literature review by the OMERACT Ultrasound Group," we must take issue with some of the statements published.

The authors state that "B-lines consist of 'comet tails'... generated by the reflection of the [lung] US beam from thickened subpleural interlobar septa." Actually, the generation of ultrasound (US) artifacts mainly depends on the high difference in acoustic impedance that the US beam encounters when it crosses surfaces with a different density. US scanner machines are calibrated at a constant sound speed of about $1500 \mathrm{~m} / \mathrm{s}$, but propagation velocity in air is only $330 \mathrm{~m} / \mathrm{s}$ (in the lung, slightly increased to $440 \mathrm{~m} / \mathrm{s}$ owing to presence of parenchyma): for this reason more than $96 \%$ of the US beam is reflected at tissue-chest wall/air-lung interface, resulting in hyperechoic pleural line and US artifacts ${ }^{2}$. In particular, B-lines, appearing as parallel hyperechogenic striae extended for the entire US image, result from different acoustic impedance between liquid film trapped in gas microbubbles and/or fibrous edema areas crossed by the US beam. That is why B-lines are also found in the bowel loops ${ }^{3}$ and in the residual cavity of the postpneumonectomy space ${ }^{4}$. On the other hand, "comet tails," running out in a few $\mathrm{mm}$ of depth in a triangular shape, derive from solid structures such as crystals (e.g., cholesterin, calcium) or pleural thickenings. Therefore, "B-lines" and "comet tails" are different in shape and content.

Number of B-lines may increase in all those pathological pleuropulmonary conditions in which the proportion of air/liquid film changed: heart failure, adult respiratory distress syndrome, pneumothorax and hydropneumothorax, fibrosis, emphysema, exacerbations of chronic obstructive pulmonary diseases, nephrotic syndrome, and lymphagitis ${ }^{5,6,7}$. This type of US artifact is not a pathognomonic index of fibrosis: for this reason it is not possible to assess the presence of an initial interstitial lung disease in systemic sclerosis ( $\mathrm{SSc}$ ) only by detecting and/or counting B-lines. Recently we studied patients with a histological diagnosis of fibrosis, performing both transthoracic US (TUS) and an intraoperative ultrasound examination during video-assisted thoracic surgery (VATS). Generation of B-lines artifacts did not occur in our intraoperative examination in VATS-US, despite the presence of B-lines in TUS. Indeed, in areas with pulmonary fibrosis (PF), only a thicker hyperechoic line compared to the nonfibrotic lung was visible, in the absence of any other artifact ${ }^{8,9}$. Unlike TUS, the VATS-US approach is not limited by differences in acoustic impedance, because the probe is directly in contact with the lung (Figure 1). This, therefore, confirms how the high difference in acoustic impedance between chest wall and air influences the visualization of the pleurae and the lungs during TUS, and consequently generates artifacts such as B-lines.

The number of B-lines also depends on the probe used, the degree of total gain compensation, and tissue harmonic and electronic beam focusing. High frequency probes (linear probe, $8-12.5 \mathrm{MHz}$ ) reduce the number of artifacts; sectorial probes $(2-3.5 \mathrm{MHz})$ create a folding in the US beam that increases the number of artifacts. The types of probes used in the studies considered by the review authors vary; they are an important cause of bias in detection/count of B-lines.

Ultimately, TUS can at best explore no more than $70 \%$ of the most superficial lung pleura facing the chest surface, where it is not obscured by bones or other structures, such as the parietal mediastinal pleura. Although this limits the effectiveness of the TUS versus high-resolution computed tomography (HRCT), PF in SSc tends to originate at the posterior and basal pulmonary fields, where TUS exploration is not critically impaired. Pleural line thickness assessed by TUS displays good concordance with HRCT patterns, indicating lung fibrosis and changes attributable to its progression ${ }^{10}$. In this context TUS may become a useful complementary tool in well-trained hands for indicating timely HRCT assessment of PF in SSc in early diagnosis and followup.

CARLA MARIA IRENE QUARATO ${ }^{\circledR}$, MD, Ospedali Riuniti di Foggia, Department of Respiratory Disease, Foggia; VALERIA VERROTTI DI PIANELLA 10 , MD, IRCCS (Institute for Research and Health Care) Ospedale Casa Sollievo della Sofferenza, Department of Pediatrics, San Giovanni Rotondo; MARCO SPERANDEO, MD, IRCCS Ospedale Casa Sollievo della Sofferenza, Unit of Interventional and Diagnostic Ultrasound of Internal Medicine, San Giovanni Rotondo, Puglia, Italy. Address correspondence to Dr. C.M. Quarato, Ospedali Riuniti di Foggia, Department of Respiratory Disease, Viale degli Aviatori 1, Foggia 71100, Italy.E-mail: c.quarato@libero.i

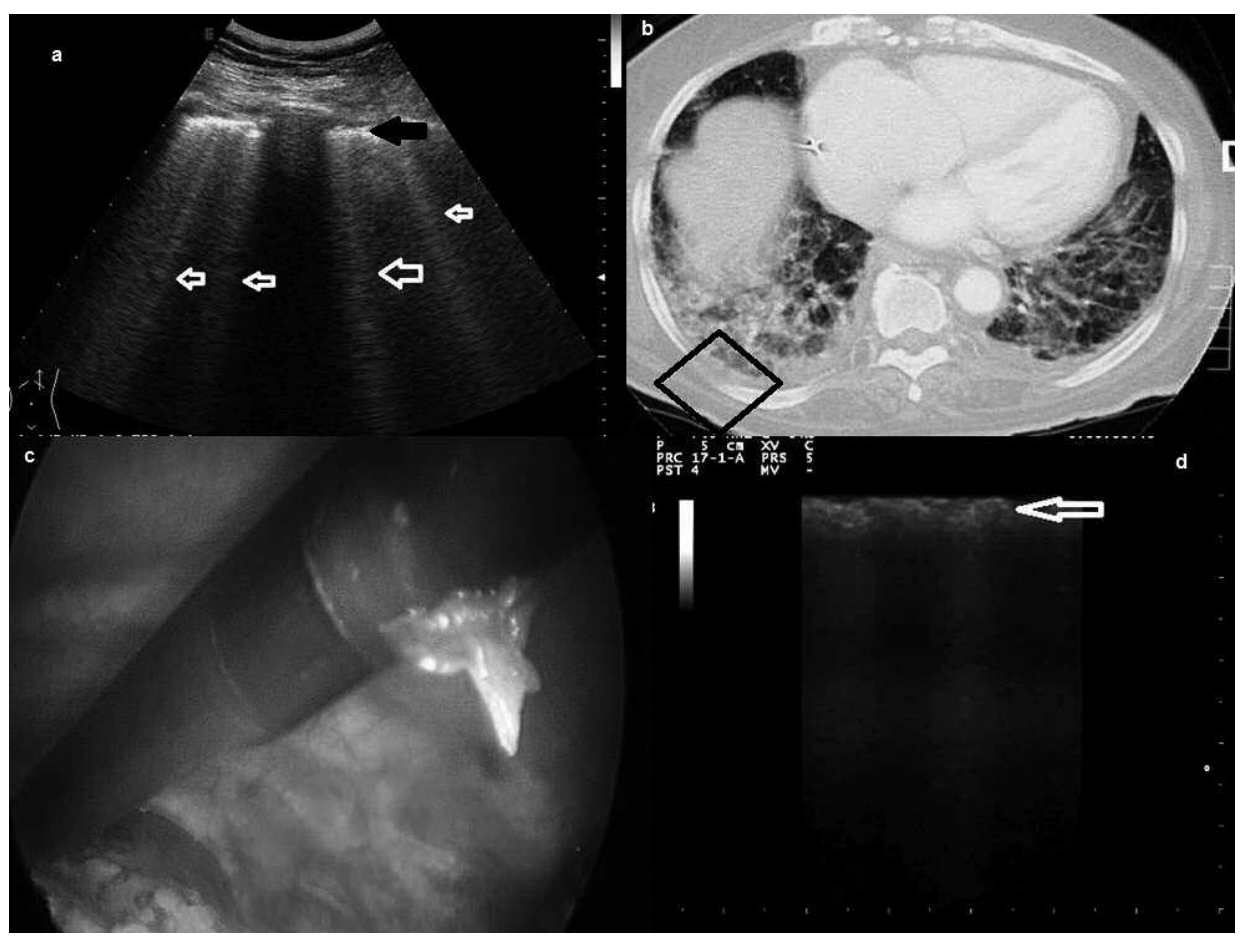

Figure 1.A. Transthoracic ultrasound (TUS) with convex probe $(5 \mathrm{MHz})$ showing irregular thickening $(>3 \mathrm{~mm})$ of the hyperechoic pleural line (black arrow) and B-lines artifacts (white arrows) below it. B. Computed tomography axial scan of the same image visualized at TUS (black box). C. VAT intraoperatory US in the same patient with fibrosis. D. Intraoperatory US image with linear probe $(10 \mathrm{MHz})$ showing increased thickness of pleural surface (white arrow) in the absence of B-lines below. VAT: video-assisted thoracic 


\section{REFERENCES}

1. Gutierrez M, Soto-Fajardo C, Pineda C, Alfaro-Rodriguez A, Terslev L, Bruyn G, et al. Ultrasound in the assessment of interstitial lung disease in systemic sclerosis. A systematic literature review by the OMERACT Ultrasound Group. J Rheumatol 2019 Jul 1 (E-pub ahead of print).

2. Sperandeo M, Rotondo A, Guglielmi G, Catalano D, Feragalli B, Trovato GM. Transthoracic ultrasound in the assessment of pleural and pulmonary diseases: use and limitations. Radiol Med 2014;119:729-40.

3. Wilson SR, Burns PN, Wilkinson LM, Simpson DH, Muradali D. Gas at abdominal US: appearance, relevance, and analysis of artifacts. Radiology 1999;210:113-23.

4. Cavaliere F, Zamparelli R, Soave MP, Gargaruti R, Scapigliati A, De Paulis S. Ultrasound artifacts mimicking pleural sliding after pneumonectomy. J Clin Anesth 2014;26:131-5.

5. Sperandeo M, Carnevale V, Varriale A. Response to pleuro-pulmonary US examination artifacts: "error in images." Ultrasound Med Biol 2010;36:356-357.

6. Zhang Y, Li J, Yang J, Zhan Y, Chen J. Lung ultrasonography for the diagnosis of 11 patients with acute respiratory distress syndrome due to bird flu H7N9 infection. Virol J 2015;12:176.

7. Marino F, Martorano C, Tripepi R, Bellantoni M, Tripepi G, Mallamaci F, et al. Subclinical pulmonary congestion is prevalent in nephrotic syndrome. Kidney Int 2016;89:421-8.

8. Tinti MG, Frongillo E, Sperandeo M. Lung fissures detection with transthoracic ultrasound. Chest 2018;154:453-5.

9. Sperandeo M, Frongillo E, Dimitri LMC, Simeone A, De Cosmo S, Taurchini M, et al. Video-assisted thoracic surgery ultrasound (VATS-US) in the evaluation of subpleural disease: preliminary report of a systematic study. J Ultrasound 2019 Mar 23 (E-pub ahead of print).

10. Sperandeo M, De Cata A, Molinaro F, Trovato FM, Catalano D, Simeone A, et al. Ultrasound signs of pulmonary fibrosis in systemic sclerosis as timely indicators for chest computed tomography. Scand J Rheumatol 2015;44:389-98.

First Release October 15 2019; J Rheumatol 2020;47:1; doi:10.3899/jrheum.190823 